\title{
Gasometría arterial ambulatoria. Recomendaciones y procedimiento
}

\author{
Arturo Cortés-Telles, * Laura Graciela Gochicoa-Rangel, ${ }^{*}$ Rogelio Pérez-Padilla, \\ Luis Torre-Bouscoulet ${ }^{*} \times$

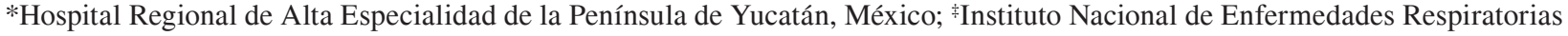 Ismael Cosío Villegas, Ciudad de México.
Trabajo recibido: 10-X-2016; aceptado: 10-XI-2016

\begin{abstract}
RESUMEN. La gasometría arterial es una prueba que permite analizar, de manera simultánea, el estado de oxigenación, ventilación y ácidobase de un individuo. Es útil para evaluar la respuesta a las intervenciones terapéuticas, farmacológicas y no farmacológicas. Asimismo, proporciona información sobre la gravedad y evolución de padecimientos previamente conocidos que involucran anormalidades en el intercambio gaseoso. El presente documento tiene como propósito brindar un panorama del procedimiento estándar vigente a nivel internacional para la toma de una gasometría arterial ambulatoria. Toma como referencia las recomendaciones emitidas por la Academia Americana de Cuidados Respiratorios (AARC, por sus siglas en inglés) en el año 2013.
\end{abstract}

Palabras clave: Gasometría arterial, procedimiento, recomendaciones.

ABSTRACT. Arterial blood gas analysis is a test that is recommended for evaluating a patient's acid-base disorders, ventilatory and oxygenation abnormalities. Is useful to follow-up a patient's response to any pharmacological and non-pharmacological interventions. Also, to analyze the severity and progression of any previous disease that has been evaluated for gas exchange abnormalities. This manuscript has the purpose to provide an overview of the current international recommendations on blood gas analysis according to the latest recommendations provided by the American Academy of Respiratory Care published in 2013.

Key words: Arterial blood gas analysis, procedure, recommendations.

\section{INTRODUCCIÓN}

La gasometría arterial (GA) es una prueba que permite analizar, de manera simultánea, el estado ventilatorio, el estado de oxigenación y el estado ácido-base. ${ }^{1} \mathrm{Se}$ realiza en una muestra de sangre arterial; no obstante, en circunstancias especiales, también se puede realizar en sangre venosa periférica o sangre venosa mezclada. ${ }^{2,3}$ En este documento se hará referencia a la GA que se realiza en pacientes estables que acuden a un laboratorio de fisiología respiratoria. ${ }^{4}$ El procedimiento que se describe a continuación forma parte de la metodología estandarizada que utilizamos en nuestro centro para la realización de la GA.

La GA proporciona mediciones directas de iones hidrógeno $(\mathrm{pH})$, presión parcial de oxígeno $\left(\mathrm{PaO}_{2}\right)$, presión parcial de dióxido de carbono $\left(\mathrm{PaCO}_{2}\right)$ y saturación arterial de oxígeno $\left(\mathrm{SaO}_{2}\right)$. Además, con el respaldo tecnológico apropiado, se puede medir la concentración de carboxihemoglobina $(\mathrm{COHb})$ y metahemoglobina (MetHb). La concentración de bicarbonato y el exceso de base efectivo no son medidos de manera directa, son valores calculados. ${ }^{2}$ Algunos gasómetros miden también electrolitos séricos, lactato, glucosa, entre otros aniones y ácidos débiles. ${ }^{5}$

\section{INDICACIONES Y CONTRAINDICACIONES}

La GA es el estándar de oro para diagnosticar anormalidades en el intercambio gaseoso y del equilibrio ácido-base. ${ }^{1}$ La GA es de utilidad en la evaluación de pacientes críticamente enfermos o pacientes estables con enfermedades respiratorias crónicas. En este último grupo es especialmente útil para analizar la necesidad de prescribir oxígeno suplementario o ventilación no invasiva en caso de insuficiencia respiratoria crónica. La GA también ayuda en el seguimiento de pacientes que han recibido intervenciones de diversas índoles, 
farmacológicas y no farmacológicas (v.gr. ejercicio), para conocer el efecto de las mismas.

Algunas contraindicaciones para realizar una GA incluyen: a) prueba modificada de Allen negativa; es decir, ausencia de circulación colateral; b) lesión o proceso infeccioso en el sitio de punción, c) ausencia de pulso en la zona donde se planea llevar a cabo la punción arterial, d) presencia de fístula arteriovenosa (tratamiento con hemodiálisis) en el sitio considerado para la punción y e) coagulopatía o anticoagulación con dosis medias-altas.

\section{EQUIPAMIENTO Y CONSUMIBLES PARA LLEVAR A CABO EL PROCEDIMIENTO DE GASOMETRÍA}

1. Jeringas de plástico o cristal desechables diseñadas para almacenar volúmenes entre 1 y 3 mililitros.

2. Agujas ${ }^{6}$

2.1 Se recomienda utilizar agujas hipodérmicas.

2.2 El tamaño de la aguja debe ser entre 20 y 23 Fr.

3. Anticoagulante. ${ }^{7}$

3.1 Utilizar Jeringas preheparinizadas.

3.2 En caso de no contar con dispositivos preheparinizados, cada jeringa deberá contener heparina no fraccionada $(0.1 \mathrm{~mL}$ de una solución de $1000 \mathrm{Ul} / \mathrm{mL}$ ) previo a realizar el procedimiento.

4. Soluciones antisépticas.

4.1 Clorhexidina al $2 \%$.

4.2 lodopovidona solución.

4.3 Torundas con alcohol.

5. Toda muestra de sangre obtenida fuera del laboratorio, remitida para el análisis gasométrico deberá estar adecuadamente etiquetada con al menos dos de los siguientes:

5.1 Nombre completo del paciente y fecha de nacimiento.

5.2 Cama del paciente (pacientes hospitalizados).

5.3 Registro hospitalario.

5.4 Fecha y hora de la toma de muestra.

5.5 Tipo de sangre: arterial, venosa periférica, venosa mezclada, capilar.

5.6 Fracción inspirada de oxígeno $\left(\mathrm{FiO}_{2}\right.$, idealmente aire ambiente; es decir, 0.21).

5.7 Temperatura corporal del paciente durante la toma de la muestra.

6. Gasas estériles.

7. Contenedor de desechos resistente a punzocortantes.

8. Guantes estériles.

9. Equipo de protección ocular.

10. Dispositivo descansa-brazo.

11. Anestésico tópico.
11.1 Lidocaína simple en solución al 2\% (en caso de instalar un catéter arterial).

\section{INSTRUCCIONES PARA EL PACIENTE PREVIO A LA TOMA DE LA MUESTRA}

1. El paciente debe evitar realizar ejercicio intenso antes del procedimiento.

2. El paciente debe evitar fumar al menos 2 horas antes de la prueba.

3. No se requiere de ayuno para la toma de la muestra.

4. No debe suspender medicación de base.

5. El paciente debe estar hemodinámicamente estable.

\section{PREPARACIÓN DEL ANALIZADOR DE GASES ANTES DE LA PRUEBA}

1. Todos los componentes (mangueras, sensores, conectores, etc.) deben ser ensamblados, de acuerdo con las instrucciones del fabricante.

2. Realizar mantenimiento preventivo acorde a los lineamientos del fabricante.

3. Se debe encender el equipo y dar tiempo suficiente para el calentamiento del mismo.

4. Calibración de gases y buffers (amortiguadores) con grado médico trazado acorde a los lineamientos internacionales.

5. El material de calibración debe reunir los requisitos reconocidos y estandarizados por organizaciones nacionales.

5.1 Los materiales de calibración deben ser etiquetados con fecha de recepción, uso y caducidad.

5.2 Todo nuevo material de calibración debe ser validado o verificado.

5.3 Debe realizarse un control de calidad para cada nuevo lote de calibración.

6. Ejemplo para calibración a dos puntos.

6.1 Verificar la temperatura del analizador, debe estar a $37 \pm 0.1^{\circ} \mathrm{C}$.

6.2 Verificar la presión barométrica del analizador; debe cotejarse de acuerdo con la altitud en la que se labora.

6.3 Mostrar la lectura de la presión barométrica en el analizador.

6.4 Iniciar una calibración a dos puntos.

6.5 Purgar gases de calibración y buffers (amortiguadores).

6.6 La calibración 1 se realiza utilizando un pH 7.348 y una mezcla de gas que contenga $\mathrm{O}_{2}$ al $20 \%$ y $\mathrm{CO}_{2}$ al $5 \%$.

6.7 La calibración 2 se realiza utilizando un $\mathrm{pH}$ 6.840 y una mezcla de gases que contenga $\mathrm{O}_{2}$ al $0 \%$ y $\mathrm{CO}_{2}$ al $10 \%$. 


\section{CALIBRACIÓN O VERIFICACIÓN DEL GASÓMETRO}

1. Control de calidad interno. ${ }^{1}$

1.1 Establecer el promedio con desviación estándar (DE) para cada componente $\left(\mathrm{pH}, \mathrm{PaCO}_{2}\right.$, $\mathrm{PaO}_{2}$ ) en cada nivel para el nuevo número de lote del material comercial para el control de calidad.

1.1.1 Analizar un número adecuado de muestras para el nuevo lote de muestreo.

1.1.2 Analizar estadísticamente los valores para cada componente en cada nivel con promedio y DE.

1.1.3 El rango aceptable para cada componente debe ser definido previamente y consistente con las necesidades para identificar anormalidades clínicas.

1.2 Los datos del control de calidad para cada lote comercial deben ser reportados mensualmente $y$ en conjunto de forma anual.

1.3 El director o encargado del laboratorio definirá el rango aceptable para el control de calidad basado en los datos estadísticos o criterios médicos.

1.4 El control de calidad debe ser analizado cada ocho horas.

1.4.1 La frecuencia de cada control y los niveles de calibración dependerán de las recomendaciones del fabricante.

1.4.2 Se debe realizar como mínimo una calibración a un nivel cada ocho horas y a dos niveles cada 24 horas.

1.5 Cuando una medición sobrepasa el promedio con 2 DE se genera una alerta y la calibración debe repetirse.

1.6 Se genera un aviso "Calibración fuera de control» cuando:

1.6.1 Una medición excede el promedio con 3 DE.

1.6.2 Dos mediciones consecutivas exceden el promedio con $2 \mathrm{DE}$.

1.6.3 Cuatro mediciones consecutivas exceden el promedio con $1 \mathrm{DE}$ en la misma dirección.

1.6.4 Diez mediciones consecutivas caen al mismo lado del promedio.

1.7 Con la presencia de un aviso "Calibración fuera de control», el equipo debe someterse a mantenimiento antes de procesar una nueva muestra

1.8 Mantener los registros del control de calidad al menos por dos años (o el tiempo que lo defina las agencias reguladoras)

\section{PREPARACIÓN DEL PACIENTE PARA LA PRUEBA}

1. El técnico que ejecuta la prueba debe recibir y presentarse ante el paciente.

2. Confirmar la orden emitida por el médico solicitante para la ejecución de la prueba, cerciorándose que se trate del paciente (nombre completo y fecha de nacimiento).

3. Explicar al paciente de forma clara y explícita el objetivo de la prueba. Puede emplearse la frase: «La gasometría arterial es una prueba que sirve para medir la cantidad de oxígeno que hay en la sangre. Para ello es necesario tomar una muestra de sangre mediante la punción de una arteria del brazo».

4. Una vez explicado el procedimiento a realizar se debe obtener la firma del consentimiento bajo información para llevar a cabo la prueba.

5. Todo personal capacitado que realice una GA deberá conocer los riesgos del procedimiento y las precauciones que deben tomarse para minimizarlos.

6. Por cuestiones de comodidad, la prueba se debe realizar con el paciente sentado.

\section{EJECUCIÓN DE LA PRUEBA}

1. Se puede obtener la muestra sanguínea de la arteria femoral, humeral o pedia; no obstante, el sitio más común es la arteria radial. Exceptuando condiciones que dificulten la toma de la muestra, se recomienda la arteria radial de la extremidad no dominante.

2. Colocar la extremidad en dorso flexión (ángulo de 45 grados) sobre un respaldo plano.

3. Realizar la Maniobra de Allen modificada con el objetivo de conocer si las arterias radial y cubital son permeables.

3.1 Ejecución de la maniobra de Allen modificada: solicitar al paciente que realice varias maniobras de apertura y cierre de la mano que será sometida a la toma de muestra. El personal que realice el procedimiento deberá realizar presión en las arterias radial y cubital con el objetivo de obstruir el flujo sanguíneo. Indicar al paciente que mantenga abierta la palma de la mano e inmediatamente liberar la presión de la arteria cubital. Observar el retorno de la coloración habitual que no debe exceder a 10 segundos y ser considerada como prueba positiva para la presencia de adecuada circulación colateral (figura 1).

3.2 Al confirmar la presencia de una adecuada circulación colateral, se lleva a cabo la desinfección del área (2 centímetros cuadrados) donde se realizará la punción arterial empleando solu- 

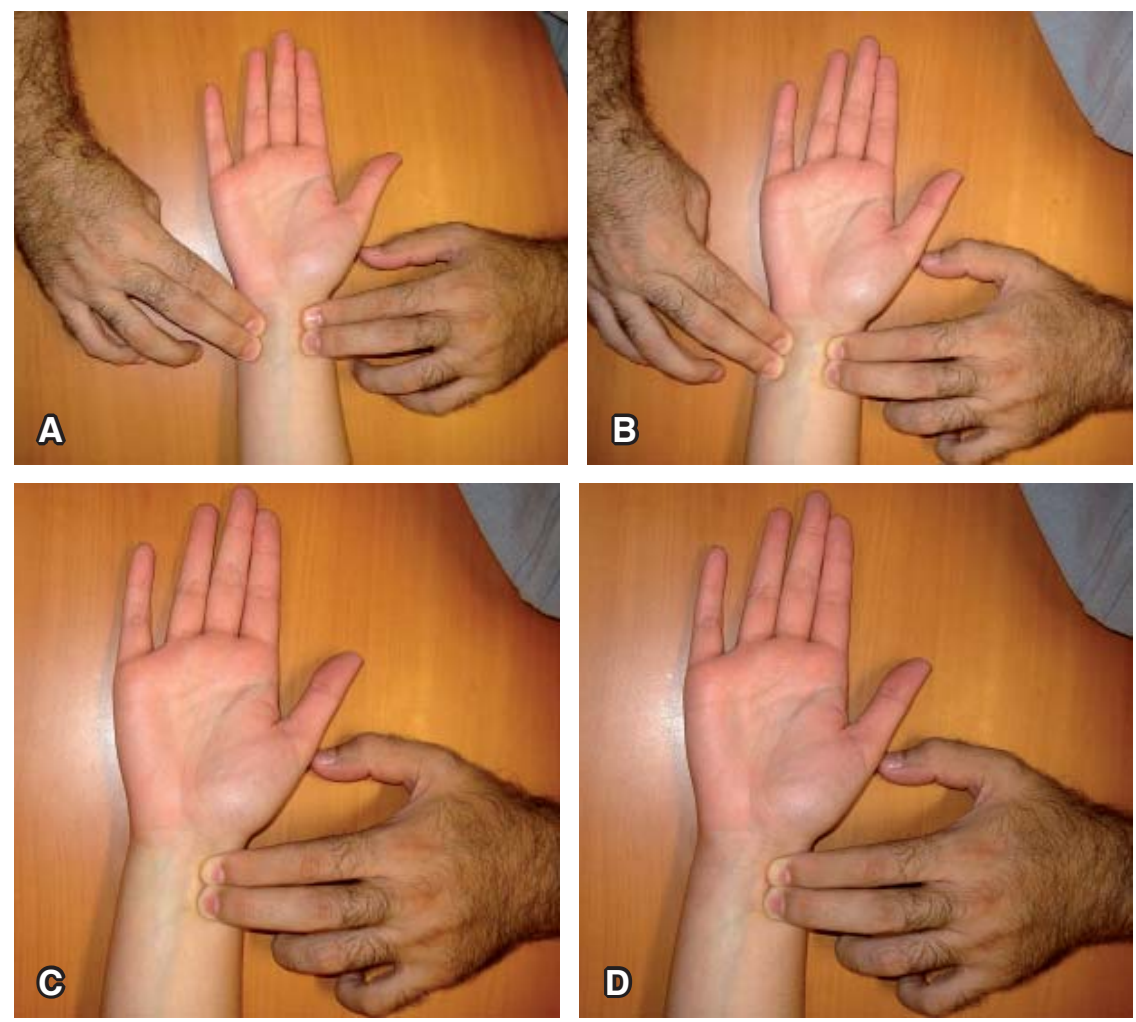

\section{Figura 1.}

Maniobra de Allen. Panel A: inicia la compresión de las arterias radial y cubital. Panel B: cambio en coloración (palidez) derivado de la oclusión. Panel C: retiro de la compresión en arteria cubital manteniendo oclusión de arteria radial. Panel D: retorno de la coloración normal en el sitio de irrigación de la arteria cubital, prueba positiva para circulación colateral. ciones antisépticas (iodopovidona en solución o clorhexidina al $2 \%$ ) durante 2 minutos.

3.3 El empleo de anestésico local es opcional. No obstante acorde con un estudio publicado, este último no es recomendable. ${ }^{8}$

4. En caso de que el paciente utilice oxígeno suplementario, éste deberá ser suspendido por al menos 20 minutos previo a la toma de muestra. En caso de que el paciente presente síntomas al retirar el oxígeno se deberá notificar al director médico del laboratorio para la mejor toma de decisión relacionada con el procedimiento.

5. El personal encargado del procedimiento deberá cerciorarse que las jeringas preheparinizadas se encuentren debidamente empaquetadas. En caso de jeringas no preheparinizadas, deberá lubricar el contenedor de la jeringa empleando heparina 0.1 $\mathrm{mL}$ (dilución 1:1,000 UI/mL). ${ }^{7}$

6. Localizar el sitio de punción palpando el pulso de la arteria.

7. Mientras continúa palpando el pulso, deberá utilizar la mano con mayor habilidad para llevar a cabo la punción de la arteria colocando la aguja adaptada a la jeringa con un ángulo de 45 grados en sentido rostral (contrario al flujo sanguíneo).

8. Al finalizar el procedimiento retirar la jeringa y comprimir con una gasa limpia y seca a una distancia de 1 o 2 centímetros del sitio de punción, en sentido proximal o rostral para vigilar complicaciones inmediatas. Se sugiere no comprimir directamente en el orificio del sitio de punción.

9. Se sugiere comprimir durante un tiempo de 3 minutos para minimizar las complicaciones.

10. La muestra obtenida debe ser mezclada continuamente utilizando las palmas de las manos en sentido rotatorio.

\section{PROCESAMIENTO DE LA MUESTRA}

1. Al extraer la muestra de sangre arterial deberá agitarse para lograr una mezcla homogénea con la heparina y evitar la formación de coágulos que pueden modificar los resultados.

2. El tiempo máximo de retraso para analizar la muestra obtenida en jeringas de plástico es de 30 minutos con temperaturas ambientales de $22{ }^{\circ} \mathrm{C} .{ }^{9}$ En caso que ocurran demoras mayores, la sugerencia es almacenar en envases de cristal o utilizar congelantes para su traslado. ${ }^{10}$

3. En el analizador de gases arteriales (figura 2), seleccionar la cantidad de muestra a procesar (se requiere entre 95 y $200 \mu \mathrm{L}$ para realizar un adecuado análisis). 


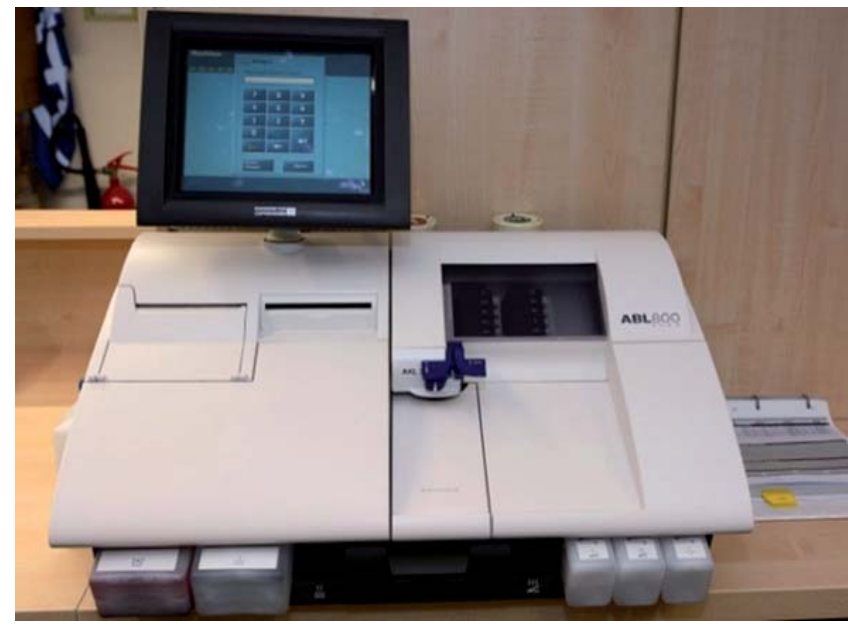

Figura 2. Gasómetro modelo ABL Flex 800 (Radiometer, Copenhagen, Denmark).

4. Previo a introducir la muestra en el receptáculo para su análisis, deberá cerciorarse que la jeringa se encuentre libre de burbujas. ${ }^{11}$

5. Abrir el receptor de muestra del analizador y colocar la jeringa para que la sangre sea aspirada. Retirar la jeringa en el momento que lo solicite el analizador y cerrar el receptor.

6. Al finalizar el aspirado de la muestra, la jeringa deberá depositarse en el contenedor resistente a punzocortantes correspondiente.

7. Anotar los datos demográficos del paciente en cuestión, no olvidar la fracción inspirada de oxígeno que en condiciones ambientales es de 0.21 (21\%).

8. Registro de la temperatura corporal. Cada laboratorio debe contar con un lineamiento para ajustar los resultados de la gasometría a la temperatura corporal del paciente. Pueden existir errores en los resultados de la muestra durante el procesamiento debido a la inclusión de un registro aleatorio de la temperatura. No hay datos disponibles que cuantifiquen la diferencia entre la entrega y consumo de oxígeno a temperaturas diferentes de $37^{\circ} \mathrm{C}$. No se recomienda llevar a cabo de rutina ajustes por temperatura. ${ }^{12}$ Los escenarios donde puede tener utilidad son todos los relacionados con proyectos de investigación ( $v$ gr. prueba de ejercicio cardiopulmonar).

9. Imprimir el informe verificando que los resultados sean consistentes. En caso contrario, deberá aparecer un informe en la pantalla, habitualmente en color rojo, lo cual es indicativo de un nuevo análisis.

\section{COMPLICACIONES DEL PROCEDIMIENTO DE GASOMETRÍA}

1. Espasmo de la arteria puncionada.
2. Reacciones vasovagales y síncope.

3. Hematoma (mayor riesgo con punciones múltiples no exitosas).

4. Trombosis arterial.

5. Trauma vascular (la lesión más grave ocurre al desgarrar la pared de la arteria, es un evento muy raro; no obstante, para disminuir el riesgo se debe introducir la aguja en línea recta, evitar en todo momento oscilaciones de la aguja durante el intento de puncionar la arteria).

6. Infección.

7. Reacción alérgica al anestésico.

8. Hemorragia.

9. Dolor en sitio de punción.

10. Parestesias durante o posterior a la punción (síntomas de lesión temporal del nervio correspondiente a la arteria intervenida. Se sugiere retirar la aguja y reintentar la punción).

\section{SEGURIDAD Y CONTROL DE INFECCIONES ${ }^{13,14}$}

1. Seguir las precauciones universales para la toma de muestras sanguíneas.

2. Utilizar guantes.

3. En caso de contacto con sangre, deben lavarse inmediatamente.

4. Reportar inmediatamente (al Departamento de Epidemiología Hospitalaria) accidentes ocasionados por punción no intencionada.

5. Depositar agujas, jeringas y muestras de sangre en contenedores correspondientes.

6. Trabajadores encargados del proceso de las muestras de gasometría deben recibir inmunización contra hepatitis $B$.

\section{REPORTE DE GASOMETRÍA}

El reporte básico debe incluir los siguientes componentes:

1. Nombre completo del paciente.

2. Tipo de muestra (sangre arterial o venosa) procesada.

3. Fracción inspirada de oxígeno con la que se procesó la muestra.

4. Temperatura del sujeto en el momento de la toma de la muestra.

5. Sitio de procedencia del paciente.

\section{PROCESO DE INTERPRETACIÓN BÁSICA}

La interpretación clínica de la GA se escapa de los alcances de este manuscrito remitimos al lector a do- 
cumentos complemenatrios..$^{15-18} \mathrm{La}$ interpretación debe incluir la evaluación del estado ventilatorio, ácido-base y de oxigenación de una manera ordenada y sistemática (tabla 1). Es importante registrar la temperatura del paciente, porque si la temperatura difiere de $37^{\circ} \mathrm{C}$, el estado in vivo podrá ser muy diferente al estado in vitro (al medir la gasometría). Habitualmente, los gasómetros trabajan con las muestras a $37^{\circ}$, luego entonces, sino ratifican la temperatura corporal del paciente en el momento de la toma de muestra, se modifican los valores de los gases de lo que esta pasando in vivo, particularmente, si la temperatura del paciente difiere mucho. La muestra recolectada está en un sistema hermético así que antes y después del cambio de temperatura, el contenido de $\mathrm{O}_{2}$ y $\mathrm{CO}_{2}$ es idéntico ya que no se puede escapar ninguna molécula. Adicionalmente, con el cambio de temperatura se cambia la $P_{50}$ de la sangre de la muestra y la afinidad de la hemoglobina por oxígeno. Al cambiar la saturación cambia la $\mathrm{PaO}_{2}$ también. Por ejemplo, si la afinidad por oxígeno de la hemoglobina se aumenta (desplazamiento a la izquierda por enfriamiento de la muestra de un paciente con

Tabla 1. Proceso de interpretación de la gasometría.

\section{Primero evaluar el estado ventilatorio}

Comparar $\mathrm{PaCO}_{2}$ con la normal para la altura $(31 \mathrm{mmHg}$ en México, $40 \mathrm{mmHg}$ a nivel del mar) y clasifica como normoventilación, hipoventilación o hiperventilación. ${ }^{16-17}$

\section{Evaluar el estado ácido-base}

Ver $\mathrm{pH}$ y exceso de base (BE) (o $\mathrm{HCO}_{3}{ }^{-}$en el sistema norteamericano). Un cambio en el exceso de base con relación al esperado a la altura ( 0 a nivel del mar, -4.85 a la altura de la Ciudad de México) significa un trastorno metabólico primario, o bien una compensación metabólica a un trastorno respiratorio. Si pH y HCO3- o BE cambian en la misma dirección (bajan o suben ambos) es metabólico primario en general; no obstante, si el cambio ocurre en dirección opuesta es compensación metabólica a un trastorno respiratorio.

\section{Ver límites de compensación del trastorno ácido-base}

Observe si hay una compensación apropiada al trastorno ácido-base, de lo contrario, estamos ante un trastorno del equilibrio ácido-base mixto. En este sentido, las compensaciones esperables se ven más claramente en un nomograma como el de Siggaard-Andersen, o mediante un programa computacional. También se suelen utilizar fórmulas para estimarlas y son más sencillas usando el exceso de base.

En los trastornos respiratorios agudos, el BE es el normal para la altura (no se modifica), por otro lado, en los trastornos respiratorios subagudos se suele lograr el $80 \%$ del ajuste completo.

En los trastornos metabólicos se puede aproximar al 50\% para la acidosis metabólica (mejor estimulante respiratorio) y al $30 \%$ para la alcalosis metabólica (depresor respiratorio mediocre). La compensación completa para un cambio en $6 \mathrm{mEQ} / \mathrm{L}$ de exceso de base se logran con $12 \mathrm{mmHg}$ de cambio de la $\mathrm{PCO}_{2}$. Si no hay compensación cualquiera de las dos alteraciones causarían un cambio de 0.1 unidades de $\mathrm{pH}$.

4. En enfermos graves evalúa el anion-gap (brecha aniónica AG) e impacto de aniones ocultos (si hay alteraciones de albúmina, $\mathrm{Na}+, \mathrm{Cl}$ - y electrolitos.

En la acidosis metabólica, el anion gap puede identificar aniones ocultos (lactato, acetoacetato y otros). Se espera un AG $\left(\mathrm{Na}^{+}-\mathrm{Cl}^{-}-\mathrm{HCO}^{3}-\right)=0.25^{\star}$ albúmina (en $\mathrm{g} / \mathrm{L}$, que es 10 veces la unidad tradicional $\mathrm{g} / \mathrm{dL}$ ). Cuando la albúmina está muy baja, o hay alteraciones importantes en los electrolitos ( $\mathrm{Na}, \mathrm{Cl}$, hiperfosfatemia) puede haber alteraciones ácido-base que no se detectan con el $\mathrm{BE}$ y el $\mathrm{pH}$ (cambios en unos pueden compensar otros y salir normal el BE).

\section{Evalúa la oxigenación}

\section{a) Analiza la $\mathrm{PaO}_{2}$ (definir hipoxemia)}

Para analizar la $\mathrm{PaO}_{2}$ se pueden tomar dos criterios:

1) Criterio terapéutico: el objetivo es llevar la $\mathrm{SaO}_{2}>90 \%$ y en hipoxemia crónica se usa $\mathrm{PaO}_{2}$ menor a $55 \mathrm{mmHg}$ o de $60 \mathrm{mmHg}$ si hay Cor pulmonale, policitemia o hipertensión pulmonar.

2) Mecanismo probable de hipoxemia a través del gradiente alvéolo-arterial. Lo que se busca es si el pulmón en cuestión intercambia gases (oxigena la sangre) de acuerdo con lo esperado para la edad (cae con edad), o si bien tiene una deficiencia en la oxigenación más allá de lo esperado por la edad y circunstancia. Si el gradiente es incrementado para la edad (esperable 1/3 de la edad), puede ser por cortocircuitos (shunt), trastorno V/Q o limitación a la difusión, este último típico de la altura y en ejercicio.

\section{b) En enfermos graves evalúa el estado de oxigenación tisular (hipoxia)}

Gasto cardíaco y perfusión tisular (hipoxia isquémica)

Contenido de $\mathrm{O}_{2}$ (hemoglobina, curva de disociación de hemoglobina con oxígeno) (hipoxia anémica)

Impedimento de la fosforilación oxidativa ( $v$.gr. cianuro) (hipoxia histotóxica) 
fiebre), la $\mathrm{PaO}_{2}$ medida estará más baja que in vivo. $\mathrm{Si}$ la afinidad por oxígeno se disminuye (desplazamiento a la derecha por calentamiento de la muestra en un paciente hipotérmico), la $\mathrm{PaO}_{2}$ medida en el gasómetro será mayor que la que se tiene in vivo. También es clave considerar que el análisis de la gasometría y en particular de la oxigenación, requiere que el paciente se encuentre en estado estable (steady-state). Si hay hipoventilación o hiperventilación aguda (o cambio de la $\mathrm{FIO}_{2}$, o de posición corporal, o cambio en la actividad física), no se consideran válidos los cambios gasométricos, específicamente los relacionados con el gradiente alvéolo-arterial, dado que el cociente respiratorio (RQ) es utilizado tanto en la ecuación del gas alveolar como el gradiente alvéolo arterial; por ende, ante las citadas eventualidades el RQ puede ser muy diferente de 0.8. No olvidar que ante circunstancias similares también se modifica el estado ácido-base.

\section{REFERENCIAS}

1. Davis MD, Walsh BK, Sittig SE, Restrepo RD. AARC clinical practice guideline: blood gas analysis and hemoximetry: 2013. Respir Care 2013;58(10):1694-1703. doi: 10.4187/ respcare.02786.

2. Herrington WG, Nye HJ, Hammersley MS, Watkinson PJ. Are arterial and venous samples clinically equivalent for the estimation of $\mathrm{pH}$, serum bicarbonate and potassium concentration in critically ill patients? Diabet Med 2012;29(1):32-35. doi: 10.1111/j.14645491.2011.03390.x.

3. Walkey AJ, Farber HW, O'Donnell C, Cabral H, Eagan JS, Philippides GJ. The accuracy of the central venous blood gas for acid-base monitoring. J Intensive Care Med 2010;25(2):104-110. doi: 10.1177/0885066609356164.

4. Breen PH. Arterial blood gas and $\mathrm{pH}$ analysis. Clinical approach and interpretation. Anesthesiol Clin North America 2001;19(4):885-906, x.

5. Palange P, Ferrazza AM. A simplified approach to the interpretation of arterial blood gas analysis. Breath 2009;6(1):14-22. doi: 10.1183/18106838.0601.014

6. Patout M, Lamia B, Lhuillier $\mathrm{E}$, et al. A randomized controlled trial on the effect of needle gauge on the pain and anxiety experienced during radial arterial puncture. PLoS One 2015;10(9):e0139432. doi: 10.1371/ journal.pone.0139432

7. Higgins $C$. The use of heparin in preparing samples for blood-gas analysis. MLO Med Lab Obs 2007;39(10):16-18.
8. Cortés-Telles A, Bautista-Bernal A, Torre-Bouscoulet L. Efecto de la anestesia en ungüento sobre la intensidad del dolor durante la realización de una gasometría arterial. Un ensayo abierto. Neumol Cir Torax 2012;71(4): 339-342.

9. Woolley A, Hickling K. Errors in measuring blood gases in the intensive care unit: effect of delay in estimation. J Crit Care 2003;18(1):31-37.

10. Knowles TP, Mullin RA, Hunter JA, Douce FH. Effects of syringe material, sample storage time, and temperature on blood gases and oxygen saturation in arterialized human blood samples. Respir Care 2006;51(7):732-736.

11. Lu JY, Kao JT, Chien TI, Lee TF, Tsai KS. Effects of air bubbles and tube transportation on blood oxygen tension in arterial blood gas analysis. J Formos Med Assoc 2003;102(4):246-249.

12. Shapiro BA. Temperature correction of blood gas values. Respir Care Clin N Am 1995;1(1):69-76.

13. Allegranzi B, Pittet D. Role of hand hygiene in healthcare-associated infection prevention. J Hosp Infect 2009;73(4):305-315. doi: 10.1016/j.jhin.2009.04.019

14. Garner JS. Guideline for isolation precautions in hospitals. The Hospital Infection Control Practices Advisory Committee. Infect Control Hosp Epidemiol 1996;17(1):53-80.

15. Wagner PD. The physiological basis of pulmonary gas exchange: implications for clinical interpretation of arterial blood gases. Eur Respir J 2015;45(1):227-243. doi: 10.1183/09031936.00039214.

16. Vázquez-García JC, Pérez-Padilla R. Valores gasométricos estimados para las principales poblaciones y sitios a mayor altitud en México. Rev Inst Nal Enf Resp Mex 2000;13(1):6-13.

17. Pérez-Padilla R, Vázquez-García JC. Calculation of gasometric values at different altitudes above sea level in Mexico. Rev Invest Clin 2000;52(2):148-155.

18. Kaufman DA. Interpretation of arterial blood gases (ABGs). ATS. Access date: 2016 September 21. Available from: https://www.thoracic.org/professionals/clinicalresources/critical-care/clinical-education/abgs.php

\section{$\triangle$ Correspondencia:}

Dr. Luis Torre-Bouscoulet, Instituto Nacional de Enfermedades Respiratorias Ismael Cosío Villegas. Calzada de Tlalpan 4502, colonia Sección XVI, Delegación Tlalpan, 14080, Ciudad de México. Correo electrónico: luistorreb@gmail.com

Los autores declaran no tener conflictos de intereses. 